\title{
On large deformation, damage and failure of ductile plates to blast loading
}

\author{
Ye Yuan ${ }^{1,2}$, P.J. Tan ${ }^{2 *}$ \\ ${ }^{1}$ Department of Mechanical Engineering, Imperial College London, Exhibition Road, \\ London $S W 7$ 2AZ, $U K$ \\ ${ }^{2}$ Department of Mechanical Engineering, University College London, Torrington Place, \\ London WC1E 7JE, UK
}

\section{Abstract}

This paper concerns the irreversible deformation and failure of metal plates to brief, but intense, dynamic (impulsive) load encountered in an air-blast. Hitherto, nearly all of the dynamic structural plasticity models of 'fully-clamped' plate only calculates its inelastic (mode I) deformation but have largely ignored damage and failure - exemplified by the partial and/or complete detachment (modes II*, II and III) of a plate from its support through material rupture - that occurs when the blast load is sufficiently intense. An analytical model that captures all three modes of the plate deformation is developed in this paper where emphasis shall be on providing the simplest formulation that contains all the essence of a material and deformation effect needed to quantify the problem. The proposed model adopts an energy density failure criterion of Shen and Jones (1992) to delineate the various inelastic failure modes; employs the constitutive framework of limit analysis for simplicity; accounts for the simultaneous influence of bending, membrane stretch and transverse shear through an interaction yield criterion; and, include the effects of strain rate on the material flow stress through the Cowper-Symonds relation. Accuracy of model predictions for square mild-steel plates are assessed through comparison with experimental data and results from finite element analysis compiled from the literature for a range of impulsive load intensities - they will be shown to be in good general agreement. Design maps delineating the different deformation régimes for different combinations of blast impulse and length versus thickness ratio are constructed for plates of equal mass.

Keywords: Plates, Damage, Energy density failure criterion, Progressive tearing, Strain-rate effect

\footnotetext{
${ }^{*}$ Corresponding author

Email addresses: ye.yuan1@ic.ac.uk (Ye Yuan $\left.{ }^{1,2}\right)$, pj.tan@ucl.ac.uk (P.J. Tan $\left.{ }^{2}\right)$
} 


\section{Introduction}

The demand for lighter designs is a continuing trend in structural applications and the consequential need to predict how light-weight components, composed of relatively thin ductile materials, respond to extreme load cases arising from blast and/or local impact is of increasing importance in design calculations [1-4]. Hitherto, nearly all the dynamic structural plasticity models of 'fully-clamped' ductile plate calculate only its mode I (inelastic) deformation. Here, we develop an analytical model that could predict the mode II, II* and III deformations of the ductile plate - these modes of deformation are exemplified by the partial and complete plate detachment from its support through material rupture.

Unlike a simple cantilever, the transverse deformation of a plate involves two spatial coordinates and the effects of large deflection are inevitably linked with in-plane stretching that soon dominates the development of its plastic deformation. Hence, the coupling between flexure, stretch and shear follows directly as a consequence of both the plastic flow law and of large deflections through the equations of motion [5]. In addition, the structural deformation is also influenced by second-order effects, such as rate dependent material properties. The emphasis of this paper is on modelling the structural response of a fully-clamped, monolithic plate rather than the interactions between blast wave and structure such as those modelled in $[6,7]$; and, to reduce the complexity of the analysis by providing the simplest possible model formulation that contains all the essence of a material and deformation effect needed to capture the physics of the problem.

$\begin{array}{ll}\text { Nomenclature } & \\ a & \text { half length of square plate } \\ c & \text { length of plastic hinge } \\ d & \text { distance from plate corner when complete detachment occurs } \\ h & \text { plate thickness } \\ I^{*}, \hat{I} & \text { non-dimensional impulse and impulse per unit area } \\ m=\rho h & \text { mass per unit area } \\ M & \text { bending moment } \\ M_{P}=4 \rho a^{2} h & \text { total mass of plate } \\ M_{0}, M_{d} & \text { static and dynamic fully plastic bending moment } \\ n & \text { material hardening coefficient } \\ N & \text { membrane force } \\ N_{0}, N_{d} & \text { static and dynamic fully plastic membrane force } \\ q, \dot{\epsilon}_{0} & \text { material constants for Cowper-Symonds constitutive equation } \\ Q & \text { transverse shear force } \\ Q_{0}, Q_{d} & \text { static and dynamic fully plastic transverse shear force } \\ t & \text { time } \\ T & \text { kinetic energy of one-quarter plate }\end{array}$




$\begin{array}{|ll|}T_{f} & \text { residual energy of one-quarter plate } \\ T_{0} & \text { initial kinetic energy of one-quarter plate } \\ V_{0}=\hat{I} / m & \text { initial velocity } \\ W & \text { transverse plate displacement } \\ W_{f} & \text { maximum central deflection } \\ W_{P}(t) & \text { temporal central deflection } \\ W_{S}(t) & \text { temporal plastic shear sliding at support } \\ \beta & \Pi_{S}^{s} /\left(\Pi_{S}^{b}+\Pi_{S}^{m}+\Pi_{S}^{s}\right) \\ \beta_{c} & \text { critical } \beta \text { value at the transition from modes II to III' } \\ \eta=a / h & \text { half length to thickness ratio } \\ \Delta & \text { plate elongation } \\ \Delta W=W-W_{S} & \text { relative displacement } \\ \epsilon_{f} & \text { uniaxial rupture strain } \\ \dot{\epsilon}_{m} & \text { uniaxial mean strain rate } \\ \phi_{i}(x, y) & \text { mode functions } \\ \Omega & \text { plastic work absorbed per unit area } \\ \Omega_{A}, \Omega_{B} & \Omega \text { at point } A \text { and } B \\ \Omega_{c} & \text { critical plastic work absorbed per unit area } \\ \bar{\Omega}\left(\triangleq \Omega_{c} / \Omega_{c}\right), \omega_{s} & \text { state variable for ductile and shear damage } \\ \Pi & \text { total potential (strain) energy for one-quarter plate } \\ \Pi_{S}, \Pi_{P} & \text { strain energy absorbed within plate and at support } \\ \Pi_{S}^{b}, \Pi_{S}^{m}, \Pi_{S}^{s} & \text { bending, shear, membrane energy at support } \\ \rho & \text { density } \\ \sigma_{0}, \sigma_{d} & \text { static and dynamic yield strength } \\ \theta & \text { relative rotation about a straight hinge line } \\ \xi & \text { position of travelling plastic hinge line relative to centre of the plate } \\ \left({ }^{\prime}\right) & \partial() / \partial t \\ () & \partial^{2}() / \partial t^{2} \\ & \end{array}$

A considerable body of experimental work on the blast response of ductile structural components already exists. One example is the classical experiment by Menkes and Opat [8] where it was shown that the deformation of fully-clamped aluminium beams under impulsive blast load can be delineated into three distinct modes: mode I - large inelastic deformation; mode II - tensile-tearing and deformation; mode III - shear-band localisation. The same modes were also reported for circular and square plates by Teeling-Smith and Nurick [9] and Olson et al. [10], respectively. Unlike beams, however, Olson et al. [10] noted that 'tensiletearing' in square plates initiates at the mid-point of the plate boundary and detachment (via material rupture) occurs progressively - the extent of which depends on the applied impulse - along the boundary towards its corners. This 'partial tearing' along parts of the plate boundary is termed mode II* by Nurick and Shave [11] and Nurick et al. [12]. The literature also contains a number of detailed three-dimensional finite element (FE) models 
of the aforesaid that could simulate the different modes of deformation to varying degrees of accuracy; for example, by Yuan and Tan [13], Gupta and Nagesh [14], Rudrapatna et al. [15], Olson et al. [10], Ramajeyathilagam and Vendhan [16], to name a few.

Typically, analytical models of plastic deformation in ductile plates to impulsive blast load were formulated within the constitutive framework of limit analysis where, for the convenience of calculating structural response, it is assumed that the stress resultants on any section, together with the approximation that plane sections remain plane, are related to the deformation by a rigid-perfectly plastic constitutive equation. Such a simplification provides a good approximation for elastic-plastic material with negligible strain hardening since it is the asymptotic limit for stress resultants as curvature becomes indefinitely large [5]. Previous works by Jones et al. [17] and Yu and Chen [18] adopt the aforesaid approach but both neglect the influence of transverse shear in their yield condition. The effects of transverse shear on plastic yielding during modes II and III deformation were considered by Shen and Jones [19] and Yuan et al. [20] for beams and, again, by [21] for circular plates. Despite the good agreement between the predicted mode I deflection of [21] with the experimental data of $[9,22]$, there are considerable discrepancies between the predicted critical impulse at mode $\mathrm{I} \rightarrow \mathrm{II}$ transition and the experimental values of [9].

In this paper, an analytical model of a fully-clamped square plate subjected to impulsive blast load is developed which, for the first time, is able to calculate all the three modes of its deformation. Results for their experimental counter-part were previously reported by Teeling-Smith and Nurick [9] and Olson et al. [10]. The analytical model is an extension of the ones developed in $[17,18]$ to include the following additional features: (1) effects of material strain rate sensitivity; (2) large inelastic deformation with catenary actions; (3) effects of transverse shear force at the support; (4) interactions between bending, membrane and shear in the yield criterion; and (5) modelling the tear initiation and progressive ductile fracture along the support through an extension of the energy density criterion of Shen and Jones [19, 21]. Accuracy of the model predictions will be assessed by comparing to experimental data and finite-element predictions compiled from the literature.

The outline of this paper is as follows: Section 2 presents the formulation of the dynamic structural plasticity model of a fully-clamped ductile plate; comparisons between predictions by the current analytical model with experimental and numerical data compiled from the literature are made in Section 3; and, Section 4 presents design charts constructed using the analytical model that was developed.

\section{Model formulation}

In this section, we formulate a dynamic structural plasticity model, within the constitutive framework of limit analysis, for a 'fully-clamped' square plate subject to an impulsive blast load. For definiteness, damage shall refer to a state of deformation during which parts of the plate become partially detached from its support - this occurs for mode II* deformation 
$[11,12]$. By contrast, failure shall refer to a total loss of load carrying capacity by the plate following its complete detachment (or severance) from the support - this occurs for modes II and III deformation.

\subsection{Problem statement}
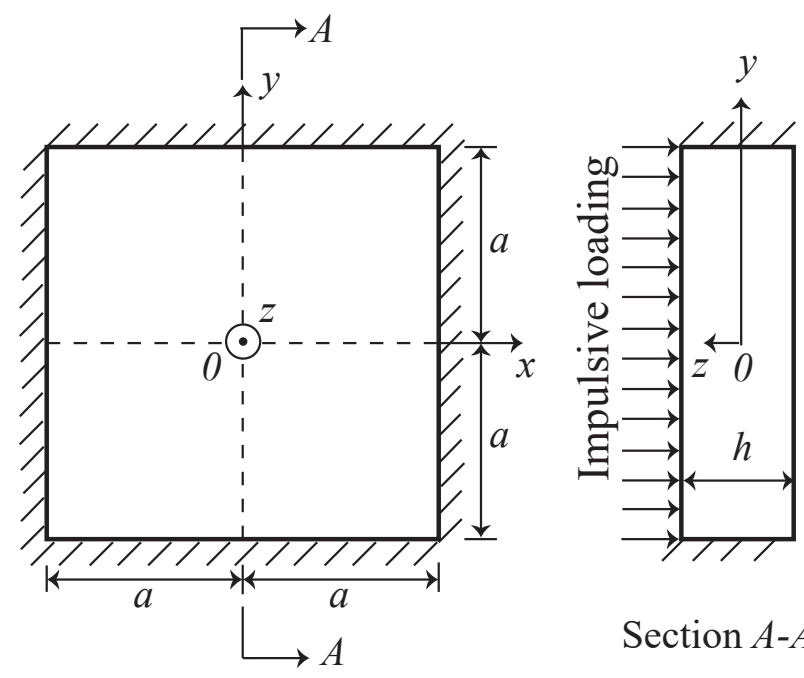

Section $A-A$

Figure 1: Schematic of a fully clamped square plate (in third-angle projection). Reflectional symmetry exists on two planes: viz. $(x=0,-h / 2 \leq z \leq h / 2)$ and $(y=0,-h / 2 \leq z \leq h / 2)$.

Consider a fully-clamped square plate of side length $2 a$ and uniform thickness $h$ subjected to a uniformly-distributed impulsive load that acts transversely to the $x$ - $y$ plane, as shown schematically in Fig. 1. Symmetry (both loading and geometric) dictates that only onequarter of the full plate needs to be modelled. Following [17-19], a rigid, perfectly-plastic material idealisation is also employed here - this simplification of the material constitutive law is needed for analytical calculations of structural response to be tractable - where elastic deformation of the plate is neglected since its transverse displacement $W \gg h$. In accordance to the Cowper-Symonds constitutive equation [23], the mean dynamic flow stress of the plate can be estimated as

$$
\sigma_{d}=\sigma_{0}\left[1+\left(\dot{\epsilon}_{m} / \dot{\epsilon}_{0}\right)^{1 / q}\right]
$$

where $\sigma_{0}$ is the static yield strength; $\dot{\epsilon}_{m}$ is the mean uniaxial strain rate; and, $\dot{\epsilon}_{0}$ and $q$ are material constants. The loading is assumed to be imparted by a blast pulse of a much shorter duration compared to the characteristic time needed by the plate to reach its maximum transverse displacement, i.e. it is a zero-period uniform momentum impulse. Hence, the plate acquires an instantaneous velocity given simply by [17-19]

$$
V_{0}=\hat{I} / m
$$


where $\hat{I}$ is the impulse per unit area acting on the plate, $m=\rho h$ is mass per unit area and $\rho$ is the plate density. The zero-period impulse can be non-dimensionalised as follows:

$$
I^{*}=\frac{V_{0}}{\sqrt{\sigma_{0} / \rho}} .
$$

\subsection{Damage and failure criteria}

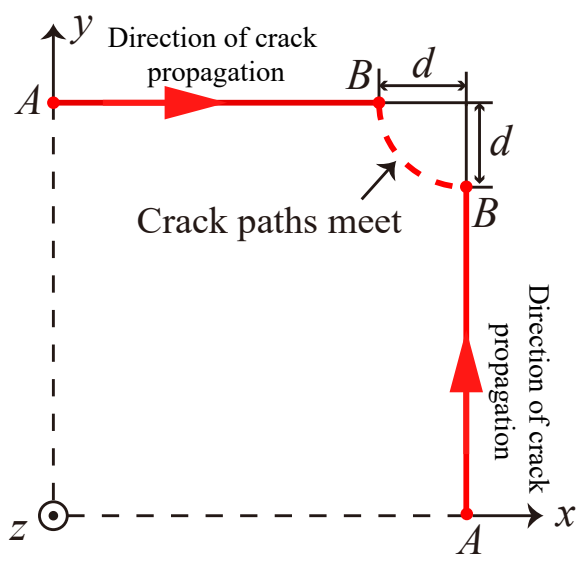

Figure 2: Direction of crack paths propagation along the plate boundary (only one-quarter of the square plate is shown).

Experiments by Nurick and Shave [11] showed the following sequence of plate deformation: (1) tearing (through material rupture) initiates simultaneously at the mid-point on each side of the square plate (points $A$ in Fig. 2); (2) as the transverse deflection of a plate increases, detachment progresses along its boundary, giving rise to two cracks propagating in opposite direction on each side; (3) the crack are deflected inwards into the plate to meet with the deflected crack from an adjoining side as it reaches a critical distance (points $B$ in Fig. 2) to circumvent the corners; and (4) complete plate detachment occurs when the two crack paths meet. A recent study by Schiano-Moriello et al. [24] - the detailed results will be reported elsewhere - have shown that the inward deflection of the crack path (as it approaches a corner) is controlled by the triaxial state of stress: in general, the direction of crack propagation follows region(s) with a zero stress triaxiality (i.e. under pure shear). A cursory post-mortem examination of the deformed test specimen (see Fig. 12c in Aune et al. [2]) shows that $d=0.097 a$; here, it suffices to assume that $d=a / 10$. To further simplify calculations, it is assumed that the time taken for the two cracks to intersect after points $B$ (see Fig. 2) is negligibly small compared to the time for a crack to propagate from $A$ to $B$.

To quantify the extent and progression of plate detachment, a damage 'measure' is proposed for each side of the plate - this is based on the energy density damage criterion proposed by Shen and Jones [19]. Symmetry of deformation dictates that only one side of the plate 
(i.e. the side of $0 \leq x \leq a-d$ and $y=a$ ) is needed to establish the energy density damage criterion which is expressed as

$$
\bar{\Omega}(x) \triangleq \frac{\Omega(x)}{\Omega_{c}} \leq 1 \quad \forall \quad 0 \leq x \leq a-d
$$

where $\Omega_{c}=\sigma_{d} \epsilon_{f} h c$ is the critical dissipation of plastic work per unit hinge length and $\epsilon_{f}$ is the uniaxial rupture strain of the plate which may be assumed to be independent of the mean uniaxial strain rate $\dot{\epsilon}_{m}$. A similar expression to Eq. 4 can be written for $\bar{\Omega}(y)$ $\forall 0 \leq y \leq a-d$. Here, $c=\alpha h$ is the width of the plastic hinge (zone) which typically ranges between $0.5 \leq \alpha \leq 1[19,21]$. It is clear that the critical impulse at mode I $\rightarrow$ II transition depends on the value of $\alpha$. Here, we use an identical $\alpha=0.6$ throughout - this was obtained through calibration to the critical impulse at mode $\mathrm{I} \rightarrow \mathrm{II}$ transition given in [11].

According to Eq. 4, initiation of material rupture, points $A$ in Fig. 2, occurs when

$$
\bar{\Omega}_{A}=\bar{\Omega}(x=0)=\frac{\Omega(x=0)}{\Omega_{c}}=1 ;
$$

whilst complete tearing follows almost immediately after the following damage criterion is met at a finite distance $d$ from the plate corner given by

$$
\bar{\Omega}_{B}=\bar{\Omega}(x=a-d)=\frac{\Omega(x=a-d)}{\Omega_{c}}=1 .
$$

The mean uniaxial strain rate $\dot{\epsilon}$ in Eq. 1 is expressed as

$$
\dot{\epsilon}_{m}=\frac{\epsilon_{f} \bar{\Omega}_{B}}{t_{f}}
$$

where time $t_{f}$ corresponds to the instant at either the point of cessation of motion (i.e. $\bar{\Omega}_{B}<1$ ) or failure (i.e. $\bar{\Omega}_{B}=1$ if complete detachment from the supports occurs).

It was noted by Nurick and Shave [11] that, in mode II $^{*}$ (i.e. $\bar{\Omega}_{A}=1$ and $\bar{\Omega}_{B}<1$ ), the plate tears away from the support resulting in 'pulling-in' of its mid-side. In the current model, however, the effects of 'pull-in' on the transverse velocity acting on the plate is neglected for simplicity. Furthermore, the model does not account for the degradation of stress resultants at the support as progressive cracking occurs along the plate boundary. Despite these simplifications, which will result in a somewhat under-prediction of the transverse plate deflection, it will be shown later that there is a good correlation between the current analytical prediction and existing experimental data.

When $\bar{\Omega}_{B}$ reaches unity, either a mode II or mode III deformation ensues. Here, we adopt a similar criterion to that proposed by Shen and Jones $[19,21]$ to delineate between a mode II 
and III deformation. Mode III deformation occurs when the state variable $\omega_{s}$ reaches unity, i.e.

$$
\omega_{s}=\frac{\beta}{\beta_{c}}=1,
$$

where $\beta$ is the ratio of the plastic work absorbed through shearing deformation to the total plastic work done by all the stress components at the entire support given by

$$
\beta=\frac{\Pi_{S}^{s}}{\Pi_{S}^{s}+\Pi_{S}^{b}+\Pi_{S}^{m}},
$$

where $\Pi_{S}^{s}, \Pi_{S}^{b}$ and $\Pi_{S}^{m}$ are, respectively, the shear strain energy, bending strain energy and membrane strain energy obtained at the support; and, $\beta_{c}$ is the critical $\beta$ value at the transition from mode II to III. Note that superscripts $s, b$ and $m$ denote shear, bending and membrane, respectively. It will be shown later that $\beta$ increases monotonically with the dimensionless impulse $I^{*}$ and the critical transition - this was obtained through calibration to the experimental data by Nurick and Shave [11] - delineating the transition between modes II and III deformation is $\beta_{c}=0.32$.

Hence, the criteria used here to delineate the different modes of plate deformation in [11] are as follows:

$$
\begin{aligned}
& \text { Mode I : } \quad \bar{\Omega}_{A}<1, \quad \bar{\Omega}_{B}<1, \quad \omega_{s}<1 \\
& \text { Mode II* }: \quad \bar{\Omega}_{A}=1, \quad \bar{\Omega}_{B}<1, \quad \omega_{s}<1 \\
& \text { Modes II : } \quad \bar{\Omega}_{A}=1, \quad \bar{\Omega}_{B}=1, \quad \omega_{s}<1 \\
& \text { Mode III : } \quad \bar{\Omega}_{A}=1, \quad \bar{\Omega}_{B}=1, \quad \omega_{s} \geq 1 \text {. }
\end{aligned}
$$

Expressions for $\bar{\Omega}_{A}, \bar{\Omega}_{B}$ and $\omega_{s}$ in Eq. 10 are derived in Section 2.4. An iterative method is used to predict the modes of deformation for a fully clamped square plate subjected to a non-dimensional impulse of $I^{*}$ (or having an initial velocity of $V_{0}$ ). For known value of $\sigma_{0}$, $\epsilon_{f}, \rho, a, h$ and $V_{0}$, and assuming an initial value for $n_{1}$, one can calculate the values of $\bar{\Omega}_{B}$ and $t_{f}$ corresponding to the cessation of plate motion (i.e. plate central velocity becomes zero), or when complete detachment occurs (i.e. $\bar{\Omega}_{B}=1$ ). This gives a new value of $n_{2}$. The above iteration on $n$ continues until $n_{i} \approx n_{i-1}$. The value of $n_{i}$ is then used to calculate the final value of $\bar{\Omega}_{A}, \bar{\Omega}_{B}, \omega_{s}$ and $t_{f}$.

\subsection{Yield condition}

Here, an interactive yield condition that combines the bending moment $M$, membrane force $N$ and the transverse shear force given by $Q[5]$

$$
\frac{|M|}{M_{d}} \sqrt{1-\frac{Q^{2}}{Q_{d}^{2}}}+\frac{N^{2}}{N_{d}^{2}}+\frac{Q}{Q_{d}}-1=0
$$


is employed to describe plastic yielding in the plate, where $M_{d}=\sigma_{d} h^{2} / 4, N_{0}=\sigma_{d} h$ and $Q_{d}=\sigma_{d} h / \sqrt{3}$ are the dynamic fully plastic bending moment, in-plane membrane force and transverse shear force, respectively. It is convenient to re-cast Eq. 11 in a non-dimensional form as follows:

$$
|\bar{M}| \sqrt{1-\bar{Q}^{2}}+\bar{N}^{2}+\bar{Q}^{2}-1=0
$$

where $\bar{M}=M / M_{d}, \bar{N}=N / N_{d}$ and $\bar{Q}=Q / Q_{d}$ are the non-dimensional fully plastic generalised stresses.

\subsection{Equations of motion}

The transverse velocity at any point $x, y(x \geq 0, y \geq 0)$ of the square plate - note that only one-quarter is modelled because of loading and geometric symmetry - may be approximated as a sum of $n$ generalised velocities and mode functions given by [25]

$$
\dot{W}(x, y, t)=\sum_{i=1}^{n} \phi_{i}(x, y) \dot{W}_{i}(t)
$$

where the partial functions $\phi_{i}(x, y)$ are admissible mode functions that satisfy the geometric boundary conditions and the temporal functions $\dot{W}_{i}(t)$ are generalised transverse velocities to be determined by the Lagrange equation of the 2 nd kind. The corresponding displacement field - this also uses the same partial functions - is given by

$$
W(x, y, t)=\sum_{i=1}^{n} \phi_{i}(x, y) W_{i}(t)
$$

The total kinetic energy at time $t$ may be written as

$$
T=\sum_{i=1}^{n_{1}} \frac{1}{2} \int_{V_{i}} \rho \dot{W}^{2}(x, y, t) \mathrm{d} V_{i}
$$

for one-quarter of the square plate which consists of $n_{1}$ number of plastic hinge zones. The potential (strain) energy is absorbed by the plastic hinge lines that separate the plastic hinge zones. Hence, the total strain energy for one-quarter of the square plate with $n_{2}$ number of plastic hinge lines, each of length $l_{i}$, can be expressed as

$$
\Pi=\sum_{i=1}^{n_{2}} \int_{l_{i}}\left(M \theta_{i}+N \Delta W \theta_{i}+Q W_{S}\right) \mathrm{d} l_{i}
$$

where $\theta_{i}$ is the relative rotation across a hinge line; $W_{S}(t)$ is plastic shear sliding distance at the support; and, $\Delta W\left(\triangleq W(x, y, t)-W_{S}(t)\right)$ is relative displacement.

Since the Lagrangian of the structural beam system is

$$
\mathcal{L}=T+\Pi
$$


the differential equations governing $W_{i}$ can be obtained by substituting Eqs. 17 and 19 into the well-known Lagrange equation of the 2nd kind as follows:

$$
\frac{\mathrm{d}}{\mathrm{d} t}\left(\frac{\partial \mathcal{L}}{\partial \dot{W}_{i}}\right)+\frac{\partial \mathcal{L}}{\partial W_{i}}=Q_{i}, \quad i=1,2, \ldots, n .
$$

Since an initial velocity is prescribed to the entire plate for impulse loading condition, the potential energy loss $E^{P}$ due to the external loading is zero; hence, the generalised force is simply

$$
Q_{i}=\frac{\partial E^{P}}{\partial W_{i}}=0 .
$$

The key to obtaining the governing equations of motion in Eq. 18 is the expressions for the total kinetic energy $T$ and strain energy $\Pi$, which will be derived later.

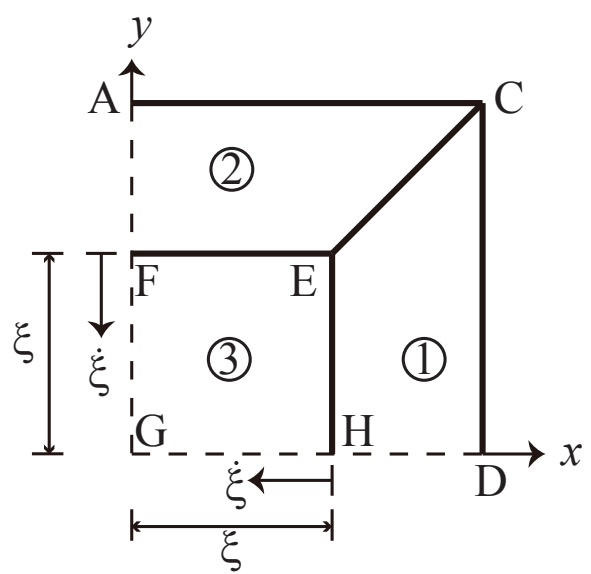

(a)

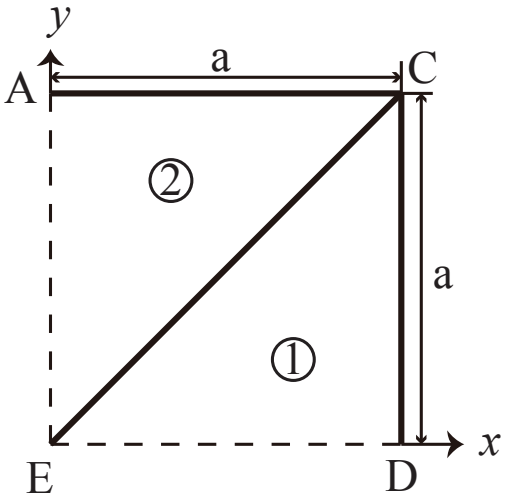

(b)

Figure 3: Deformation mechanisms for one-quarter of the square plate depending on the position of travelling plastic hinge lines [18]: (a) $0<\xi<a$ and (b) $\xi=0$.

Within the constitutive framework of limit analysis, travelling plastic hinge lines develop in a rigid, perfectly-plastic plate along the principal stress-moment directions [23]. According to the position of the travelling hinge line, there are two stages of deformation for a plate (see Fig. 3): (1) when plastic hinge lines travel towards the centre of the plate; and (2) when the plastic hinge lines reach and coalesce at the centre, leading to a final (and stationary) collapse configuration. Following [17-19, 21], it is assumed that the membrane force $\bar{N}$ is identical throughout the plastic hinge lines and transverse shear is negligible on the plastic hinge lines located in the plate interior. According to Eq. 11, the yield condition at the support (i.e. hinge lines $A C$ and $C D$ ) and in the plate interior (i.e. plastic hinge lines $C E$, $E F$ and $E H)$ are given, respectively, by

$$
\begin{aligned}
\left|\bar{M}_{S}\right|\left(1-\bar{Q}_{S}^{2}\right)+\bar{N}^{2}+\bar{Q}_{S}^{2}=1, & \text { if } & \left|\bar{M}_{S}\right|>0 \\
\bar{N}^{2}+\bar{Q}_{S}^{2}=1, & \text { if } & \left|\bar{M}_{S}\right|=0
\end{aligned}
$$


and

$$
\bar{M}_{P}+\bar{N}^{2}=1 \text {. }
$$

Note that subscripts $S$ and $P$ are used here to denote support and plate, respectively.

For the deformation mechanism in Fig. 3a, the transverse velocity profiles in zone (1), (2) and (3) are given by

$$
\begin{array}{rr}
\dot{W}_{1}(x, y, t)=\dot{W}_{S}(t)+\left[\dot{W}_{P}(t)-\dot{W}_{S}(t)\right](a-x) /[a-\xi(t)], & \text { Zone (1) } \\
\dot{W}_{2}(x, y, t)=\dot{W}_{S}(t)+\left[\dot{W}_{P}(t)-\dot{W}_{S}(t)\right](a-y) /[a-\xi(t)], & \text { Zone (2) } \\
\dot{W}_{3}(x, y, t)=\dot{W}_{P}(t), & \text { Zone (3). }
\end{array}
$$

When the travelling plastic hinge lines reach the centre of the plate (i.e. $x=0$ and $y=0$ ), the transverse velocity profiles in Fig. $3 \mathrm{~b}$ become

$$
\begin{aligned}
& \dot{W}_{1}(x, y, t)=\dot{W}_{S}(t)+\left[\dot{W}_{P}(t)-\dot{W}_{S}(t)\right](a-x) / a, \quad \text { Zone (1) } \\
& \dot{W}_{2}(x, y, t)=\dot{W}_{S}(t)+\left[\dot{W}_{P}(t)-\dot{W}_{S}(t)\right](a-y) / a, \quad \text { Zone (2). }
\end{aligned}
$$

It is worth highlighting that in Fig. 3a, the central portion of the plate is assumed to travel at a constant transverse speed $[17,18]$, i.e.

$$
\ddot{W}_{P}=0, \quad \dot{W}_{P}=V_{0},
$$

and its acceleration is solely governed by $\ddot{W}_{S}$; whereas in Fig. $3 \mathrm{~b}$, the motion of the central portion of the plate is determined by both $\ddot{W}_{P}$ and $\ddot{W}_{S}$.

Taking into account the deformation mechanisms that develop in the square plate (a quarter), its total kinetic energy (Eq. 15) and strain energy (Eq. 16) are given, respectively, by

$$
\begin{aligned}
& T=\frac{1}{2} m\left[\int_{\xi(t)}^{a} \int_{0}^{\xi(t)} \dot{W}_{1}^{2}(x, y, t) \mathrm{d} x \mathrm{~d} y+\int_{\xi(t)}^{a} \int_{\xi(t)}^{x} \dot{W}_{1}^{2}(x, y, t) \mathrm{d} x \mathrm{~d} y\right. \\
& \left.+\int_{\xi(t)}^{a} \int_{x}^{a} \dot{W}_{2}^{2}(x, y, t) \mathrm{d} x \mathrm{~d} y+\int_{0}^{\xi(t)} \int_{\xi(t)}^{a} \dot{W}_{2}^{2}(x, y, t) \mathrm{d} x \mathrm{~d} y+\int_{0}^{\xi(t)} \int_{0}^{\xi(t)} \dot{W}_{3}^{2}(x, y, t) \mathrm{d} x \mathrm{~d} y\right]
\end{aligned}
$$

and

$$
\begin{aligned}
\Pi=2 \xi(t)\left[M_{S} \theta_{1}+N\right. & \left.\Delta W_{P} \theta_{1}+Q_{S} W_{S}(t)\right]+2 \int_{\xi(t)}^{a}\left(M_{S}+N \Delta W_{1}\right) \theta_{1}+Q_{S} W_{S}(t) \mathrm{d} x \\
& +\sqrt{2} \int_{\xi(t)}^{a}\left[M_{S}+N \Delta W_{1}\right] \theta_{3} \mathrm{~d} x+\xi(t)\left(2 \theta_{1}+2 \theta_{2}\right)\left(M_{P}+N \Delta W_{3}\right)
\end{aligned}
$$

where $\Delta W_{P}=W_{P}(t)-W_{S}(t), \Delta W_{1}=W_{1}(x, y, t)-W_{S}(t)$ and $\Delta W_{3}=W_{3}(x, y, t)-W_{S}(t)$; $\theta_{1}=\theta_{2}=\Delta W_{P} /[a-\xi(t)]$ are relative rotations along the outer boundaries (fully clamped) of plastic zone (1) and (2) respectively; and $\theta_{3}=\sqrt{2} \theta_{1}$ is the change in rotation across the inclined plastic hinges that lie at the intersections of plastic zone (1) and (2). Note that the 
sum of the first two terms in Eq. 26 corresponds to the strain energy dissipated along its support, whereas the last term relates to the strain energy absorbed at the plate interior.

The parameters $M_{S}, Q_{S}, N, M_{P}$ and $\xi(t)$ in Eqs. 25 and 26 are unknowns. Recasting them in non-dimensional form, viz. $\bar{M}_{S}, \bar{Q}_{S}, \bar{N}, \bar{M}_{P}$ and $\bar{\xi}=\xi(t) / a$, they are computed as described below.

The non-dimensional position of the travelling plastic hinge line is

$$
\bar{\xi}=\int_{0}^{t} \dot{\bar{\xi}} \mathrm{d} t
$$

3 and $\dot{\bar{\xi}}$ is the non-dimensional velocity of the travelling plastic hinge line given by

$$
\dot{\bar{\xi}}=\frac{\left(\sigma_{d} / \rho a^{2}\right)\left[12\left(\bar{M}_{S}+\bar{M}_{P}\right)+24 \bar{N} \Delta \bar{W}_{P}(1+\bar{\xi})\right]+(1-\bar{\xi})^{2}(1+\bar{\xi}) \ddot{\bar{W}}_{S}}{(\bar{\xi}-1)(1+3 \bar{\xi}) \Delta \dot{\bar{W}}_{P}}
$$

where $\bar{W}_{P}=W_{P}(t) / h, \dot{\bar{W}}_{S}=\dot{W}_{S}(t) / h, \Delta \bar{W}_{P}=\Delta W_{P} / h$ and $\ddot{\bar{W}}_{S}=\ddot{W}_{S}(t) / h$. When the travelling plastic hinge lines $E F$ and $E H$ reach the centre of the plate, they coalesce with the plastic hinge line $C E$ into a single plastic hinge line so that in the subsequent motion,

$$
\dot{\bar{\xi}}=0 \quad \text { and } \quad \bar{\xi}=0 \text {. }
$$

The generalised strain rates associated with bending $\dot{\theta}_{S}$ and shear $\dot{\bar{\Delta}}$ are given by

$$
\begin{array}{r}
\dot{\theta}_{S}=\frac{\Delta \dot{\bar{W}}_{P}}{\eta(1-\bar{\xi})} \\
\dot{\bar{\Delta}}=\frac{\dot{\Delta}}{h}=\frac{\Delta \bar{W}_{P} \Delta \dot{\bar{W}}_{P}}{\eta(1-\bar{\xi})}
\end{array}
$$

where $\eta=a / h$. Normality requirements dictate that [23]

$$
\begin{array}{r}
\frac{\dot{\bar{\Delta}}}{\dot{\theta}_{S}}=\frac{1}{2} \bar{N}\left(1+\frac{1}{\sqrt{1-\bar{Q}_{S}^{2}}}\right) \\
\frac{\dot{\bar{W}}_{S}}{\dot{\theta}_{S}}=\frac{\sqrt{3}}{4}\left(\frac{2 \bar{Q}_{S}}{\sqrt{1-\bar{Q}_{S}^{2}}}-\frac{\bar{Q}_{S} \bar{M}_{S}}{1-\bar{Q}_{S}^{2}}\right)
\end{array}
$$

217 if $\bar{M}_{S}>0$, and

$$
\frac{\dot{\bar{W}}_{S}}{\dot{\bar{\Delta}}}=\frac{\sqrt{3} \bar{Q}_{S}}{\bar{N}}
$$


if $\bar{M}_{S}=0$. Substituting Eqs. 30 into 31 and 32, and re-arranging, gives

$$
\begin{array}{r}
\bar{N}\left(1+\frac{1}{\sqrt{1-\bar{Q}_{S}^{2}}}\right)=2 \Delta \bar{W}_{P} \\
\bar{Q}_{S}\left(\frac{2}{\sqrt{1-\bar{Q}_{S}^{2}}}-\frac{\bar{M}_{S}}{1-\bar{Q}_{S}^{2}}\right)=\frac{\gamma(1-\bar{\xi}) \dot{\bar{W}}_{S}}{\Delta \dot{\bar{W}}_{P}}
\end{array}
$$

218

if $\bar{M}_{S}>0$, and

$$
\frac{\bar{Q}_{S}}{\bar{N}}=\frac{\gamma \dot{\bar{W}}_{S}(1-\bar{\xi})}{\Delta \dot{\bar{W}}_{P} \Delta \bar{W}_{P}}
$$

if $\bar{M}_{S}=0$, where $\gamma=Q_{d} a / M_{d}=4 a / \sqrt{3} h$.

To calculate $\bar{M}_{S}, \bar{Q}_{S}, \bar{N}$ and $\bar{M}_{P}$, they would need to be expressed as functions of $\bar{W}_{P}$, $\bar{W}_{S}, \dot{\bar{W}}_{P}, \dot{\bar{W}}_{S}, \bar{\xi}$ and $t$ through Eqs. 20, 21, 28, 33 and 34. The temporal evolution of these parameters are obtained by solving the aforementioned equations using the 4th order Runge-Kutta method with the initial conditions of $\bar{W}_{P}=\bar{W}_{S}=\dot{\bar{W}}_{S}=0$ and $\dot{\bar{W}}_{P}=V_{0}$. When considering the influences of membrane forces and transverse shear forces as well as bending moments in the yield condition, the travelling hinge will initiate from a position which is near to the support but not from it $[19,21]$. Here, an initial plastic hinge line position of $\left.\bar{\xi}\right|_{t=\Delta t}=1-\sqrt{3 M_{0} \Delta t / \rho a^{2} h V_{0}}[18]$ over a small time step $\Delta t$ is used.

Since the bending, membrane and shear strain energy dissipated at the support are given by

$$
\begin{array}{r}
\Pi_{S}^{b}=2 a M_{S} \theta_{1} \\
\Pi_{S}^{m}=2 \xi(t) N \Delta_{P} \theta_{1}+2 \int_{\xi(t)}^{a} N \Delta W_{1} \theta_{1} \mathrm{~d} x \\
\Pi_{S}^{s}=2 a Q_{S} W_{S}(t),
\end{array}
$$

the expression for $\omega_{s}$ can be written as follows (by substituting Eq. 35 into 8 and rearranging):

$$
\omega_{s}=\frac{\beta}{\beta_{c}}=\frac{1}{\beta_{c}} \frac{\gamma \bar{Q}_{S} \bar{W}_{S}}{\eta \bar{M}_{S} \theta_{1}+4 \eta \bar{\xi} \bar{N} \Delta \bar{W}_{P} \theta_{1}+\int_{\bar{\xi}}^{1} 4 \eta \bar{N} \Delta \bar{W}_{1} \theta_{1} \mathrm{~d} \bar{x}+\gamma \bar{Q}_{S} \bar{W}_{S}}
$$

where $\bar{x}=x / a$.

The density of plastic energy dissipation at any location $0 \leq x \leq a$ on one side of the boundary is given by

$$
\Omega(x)=\left\{\begin{array}{cc}
M_{S} \theta_{1}+N \theta_{1} \Delta W_{P}+Q_{S} W_{S}(t), & 0 \leq x \leq \xi(t) \\
M_{S} \theta_{1}+N \theta_{1} \Delta W_{1}+Q_{S} W_{S}(t), & \xi(t) \leq x \leq a
\end{array}\right.
$$


Therefore the damage variables $\bar{\Omega}_{A}$ (in Eq. 5 ) and $\bar{\Omega}_{B}$ (in Eq. 6) become

$$
\bar{\Omega}_{A}=\frac{\bar{M}_{S} \theta_{1}+4 \bar{N} \theta_{1} \Delta \bar{W}_{P}+\gamma / \eta \bar{Q}_{S} \bar{W}_{S}}{4 \epsilon_{f} \alpha}
$$

and

$$
\begin{aligned}
& \bar{\Omega}_{B}=\bar{\Omega}_{A}, \bar{\xi} \geq \bar{d} \\
& \bar{\Omega}_{B}=\frac{\bar{M}_{S} \theta_{1}+4 \bar{N} \theta_{1} \Delta \bar{W}_{P}(1-\bar{d}) /(1-\bar{\xi})+\gamma / \eta \bar{Q}_{S} \bar{W}_{S}}{4 \epsilon_{f} \alpha}, \quad \bar{\xi}<\bar{d}
\end{aligned}
$$

Plate deformation ends at time $t=t_{f}$ if the motion of the plate ceases, i.e.

$$
\dot{\bar{W}}_{P}=0 .
$$

If the damage variable $\bar{\Omega}_{B}<1$ when this occurs, the plate is said to deform in either mode I or II*. Otherwise, a mode II or III deformation would develop if $\bar{\Omega}_{B}=1$.

If the plate fails before all its initial kinetic energy is expended, then it would acquire a residual kinetic energy at the point of severance from the support. Parts of this are absorbed through further plastic deformation as the plate continues to deform until it reaches a rigid permanent set whilst the remaining as translational kinetic energy. In the current model, the energy that is absorbed post severance from the support is not considered. The residual energy of the plate (one quarter) at the instant of plate severance is

$$
T_{f}=T\left(t=t_{f}\right)
$$

or, in non-dimensional form, as

$$
\bar{T}_{f}=\frac{T_{f}}{T_{0}}
$$

\section{Results and discussion}

In this section, the analytical predictions are validated against the experimental data of Nurick and Shave [11] for fully-clamped square plates. It is worth emphasizing that the blast loadings in [11] may be idealised as zero-period impulses [13, 15]. The square mildsteel plates used in [11] have dimensions of $0.089(2 a) \times 0.089(2 a) \times 0.0016(h)$, and their material properties are listed in Table 2. A flow-chart of the numerical implementation is shown in Fig. 4.

Figure 5 compares the predicted central deflection $\left(\bar{W}_{f}\right)$ of the plate to its corresponding experimental data. Numerical predictions by Yuan and Tan [13] and Rudrapatna et al. [15] were also included in the same figure for comparison. In general, there is an excellent 


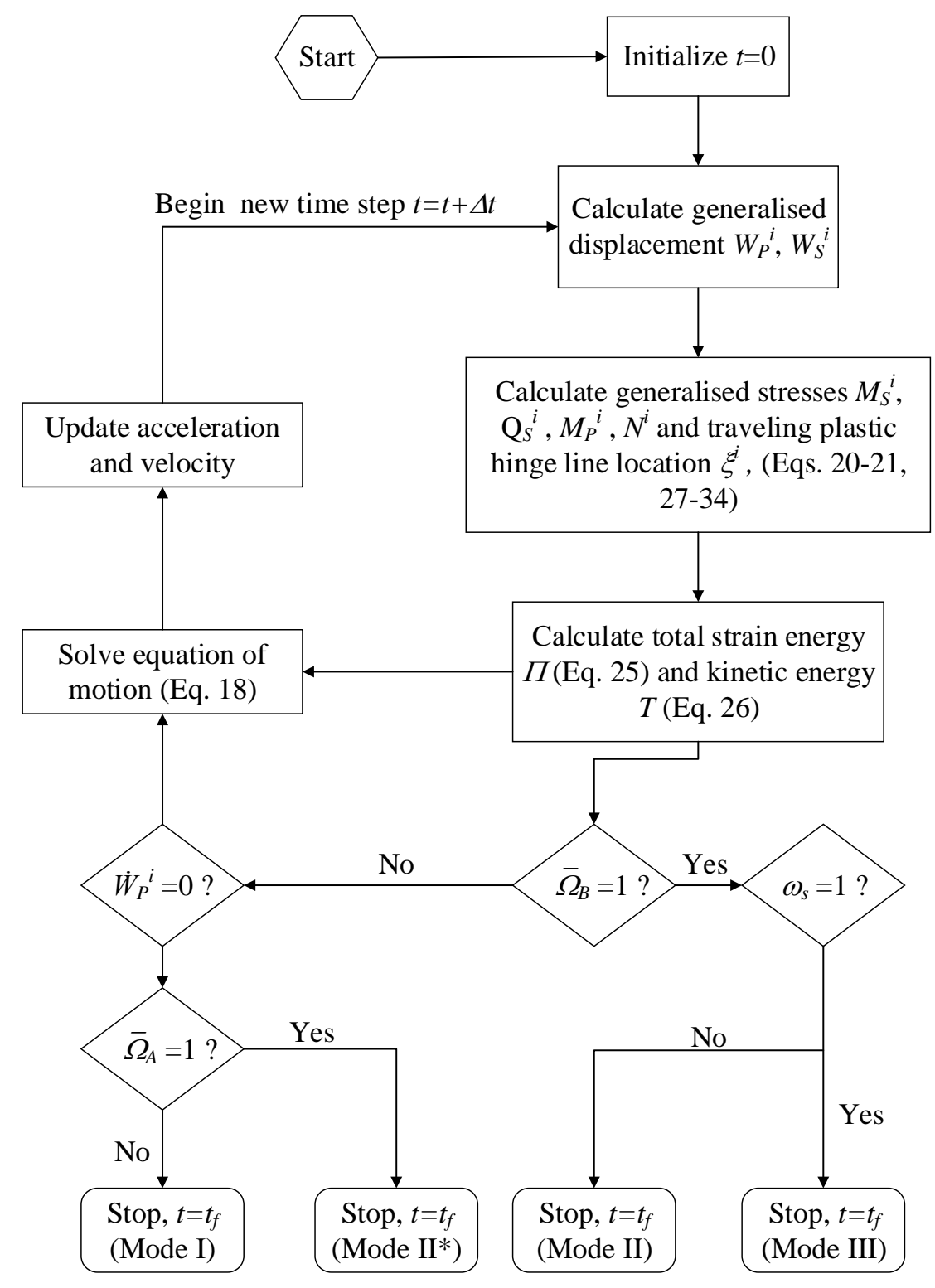

Figure 4: Flow-chart of numerical implementation for solving the analytical model described in Section 2.

agreement, between the current analytical predictions and experimental data, for plates deforming in modes I and $\mathrm{II}^{*}$. By contrast, the numerical predictions in $[13,15]$ tend to over-predict the mode I and II* deflections. This is clearly evident in Fig 6 where the analytical prediction of the temporal central plate deflection and the numerical prediction by [15] were compared to the experimental data of [11].

Figure 5 shows that the current analytical model correctly estimate a reduction in $\bar{W}_{f}$ with 
Table 2: Material properties for the mild-steel plate [11]

\begin{tabular}{ccccc}
\hline Density & Static yield stress & Rupture strain & \multicolumn{2}{c}{ Material constant } \\
$\rho\left(\mathrm{kg} / \mathrm{m}^{3}\right)$ & $\sigma_{0}(\mathrm{MPa})$ & $\epsilon_{f}$ & $\dot{\epsilon}_{0}$ & $q$ \\
\hline 7830 & 237 & 0.31 & 40.4 & 5 \\
\hline
\end{tabular}

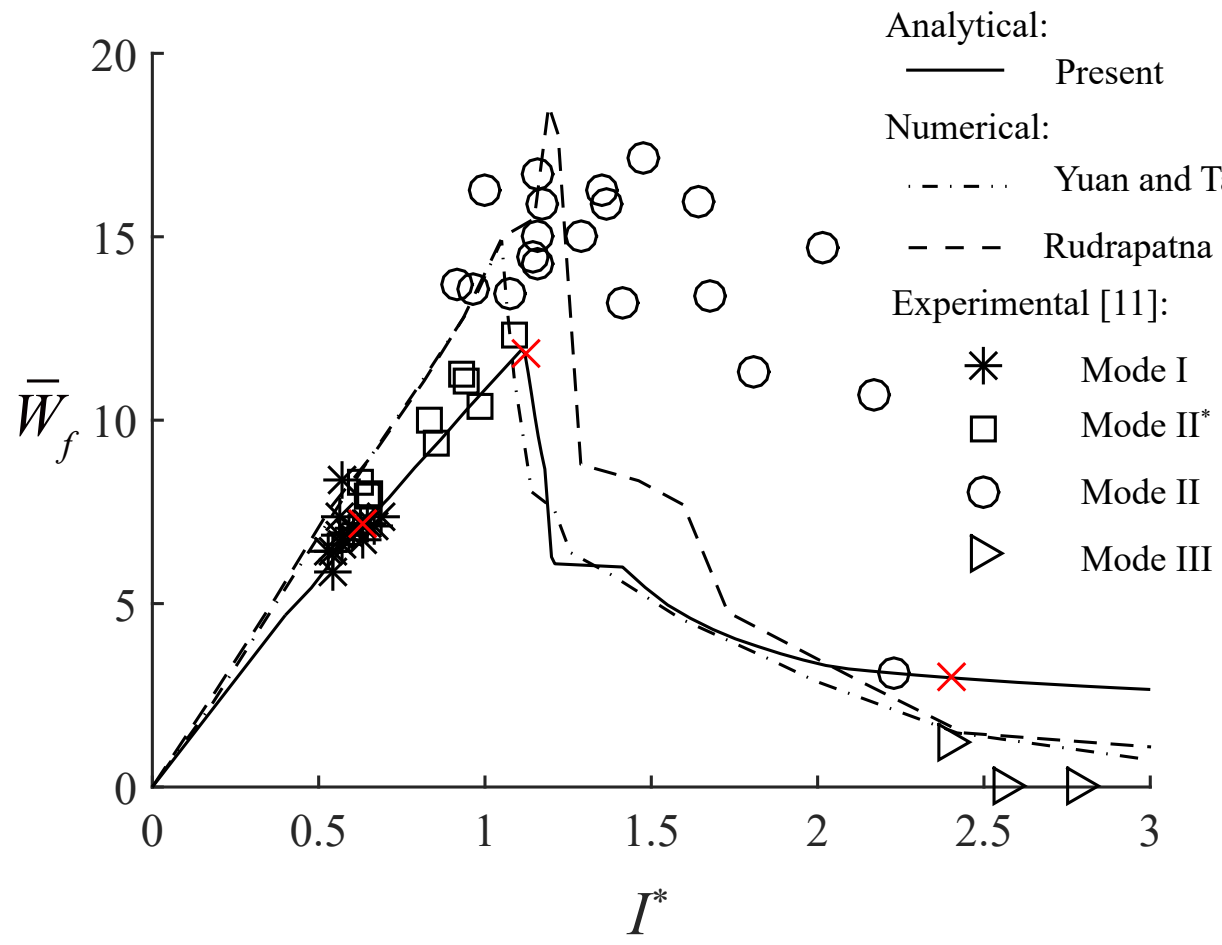

Figure 5: Comparison of the analytical central (maximum) deflection with numerical and experimental data at different levels of $I^{*}$. Each red cross indicates a transition in deformation mode predicted by the current analytical model.

increasing $I^{*}$ in mode II; in addition, they are also in excellent agreement with the predictions by Yuan and Tan [13]. The discrepancy between analytical predictions and experimental data in mode II can be attributed to the fact that the former records the central deflection just before complete detachment from the support occurs unlike in the latter. The current model over-predicts the central deflection in mode III since it assumes a constant length of plastic hinge $c=\alpha h$ following the approach of [20,26,27]. Unlike in [19], if $\alpha$ is obtained by calibration to the experimental data (mode III deflection), a better correlation between the analytical prediction and its experimental counterpart, and a monotonically decreasing of $\alpha$ with $I^{*}$, can be expected.

Table 3 compares the range of non-dimensional impulse $I^{*}$ predicted by the current model for each mode of deformation with those from $[11,13,15]$. It is clear that there are overlaps in the non-dimensional $I^{*}$ between mode I and mode $\mathrm{II}^{*}$ and between mode $\mathrm{II}^{*}$ and mode 


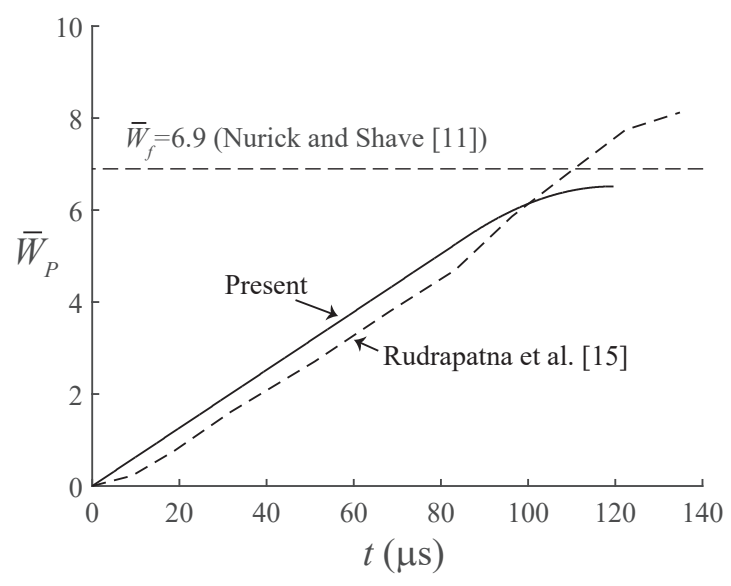

(a) $I^{*}=0.58$

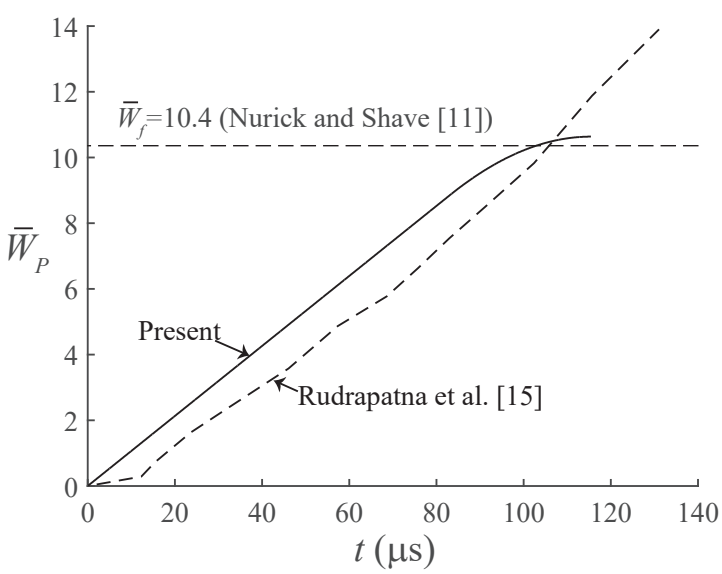

(b) $I^{*}=0.98$

Figure 6: Time history for non-dimensional central deflection of plates deforming in (a) mode I and (b) mode $\mathrm{II}^{*}[11]$.

Table 3: Range of non-dimensional impulse $I^{*}$ for each mode of deformation considered in the different studies.

\begin{tabular}{ccccc}
\hline Mode & Current model & Yuan and Tan [13] & Rudrapatna et al. [15] & Nurick and Shave [11] \\
\hline I & $0-0.62$ & $0-0.75$ & $0-0.97$ & $0.53-0.64$ \\
II $^{*}$ & $0.62-1.12$ & $0.75-0.98$ & $0.97-1.02$ & $0.62-1.09$ \\
II & $1.12-2.39$ & $0.98-2.45$ & $1.02-2.32$ & $0.92-2.39$ \\
\hline
\end{tabular}

II in the experimental data of Nurick and Shave [11]. In general, the current analytical model better predicts the range of $I^{*}$ corresponding to the different modes of deformation than the numerical ones in literature; the predicted $I^{*}$ in mode II also agrees well with the experimental data.

The 'post-detached' residual energy $\bar{T}_{f}$ for fully-severed square plates is plotted in Fig. 7. There is a good general agreement between the current analytical predictions and the numerical predictions by [15]. However both are considerably higher than the experimental data. The reason is that the loss of kinetic energy due to further plastic deformation (after complete detachment has occurred) is neglected in both the current and the numerical model of [15]. It is also evident from the figure that, beyond the transition from mode $\mathrm{II}^{*} \rightarrow \mathrm{II}$, the residual energy rises sharply, and monotonically, with increasing $I^{*}$. Consequently, even though a large impulse is generally needed to induce mode III damage during impulsive loading conditions, a significant amount of residual kinetic energy remains in the plate after it is severed.

The components of plastic work absorbed at the support through bending, membrane and 


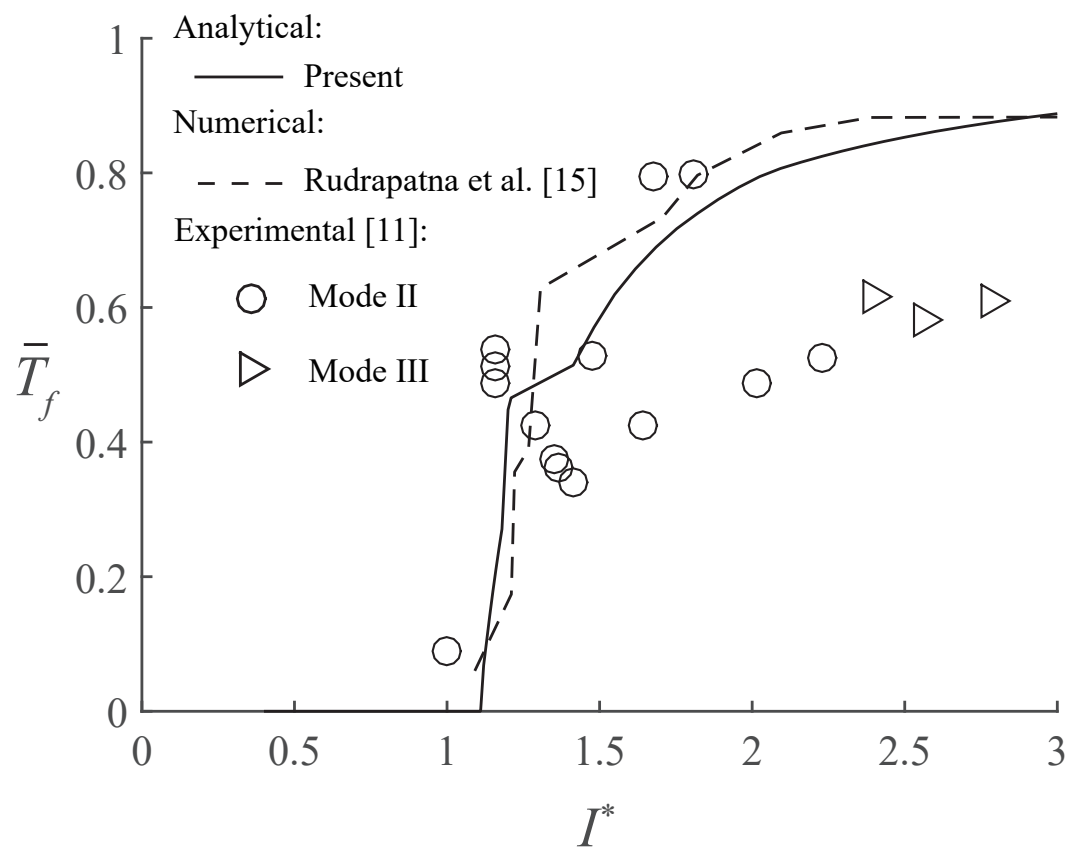

Figure 7: Non-dimensional residual energy of the fully-severed square plate.

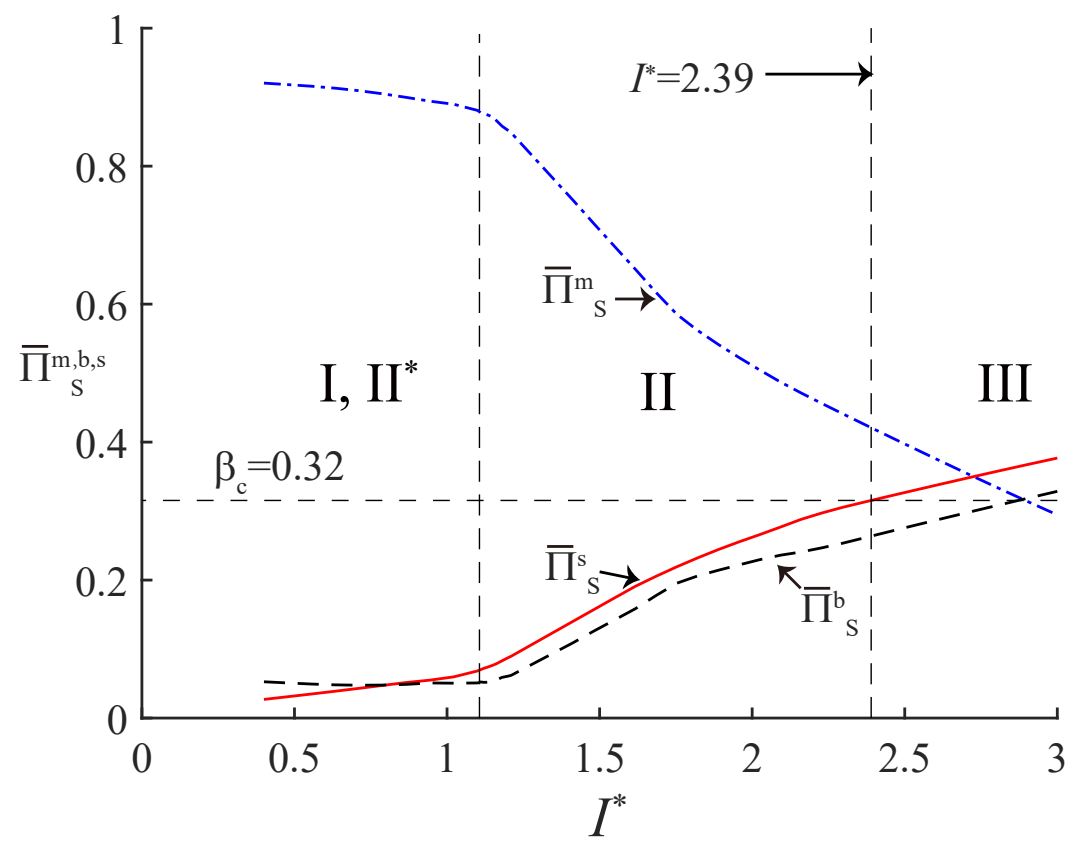

Figure 8: Components of the plastic work absorbed through bending, membrane and shear deformation at the supports.

shear deformation can be non-dimensionalised as follows:

$$
\bar{\Pi}_{S}^{b}=\frac{\Pi_{S}^{b}}{\Pi_{S}^{s}+\Pi_{S}^{b}+\Pi_{S}^{m}}, \quad \bar{\Pi}_{S}^{m}=\frac{\Pi_{S}^{m}}{\Pi_{S}^{s}+\Pi_{S}^{b}+1 \bar{B}_{S}^{m}}, \quad \text { and } \quad \bar{\Pi}_{S}^{s}=\beta=\frac{\Pi_{S}^{s}}{\Pi_{S}^{s}+\Pi_{S}^{b}+\Pi_{S}^{m}}
$$


where $\bar{\Pi}_{S}^{b}+\bar{\Pi}_{S}^{m}+\bar{\Pi}_{S}^{s}=1$. Figure 8 plots the aforesaid components of plastic work as a function of $I^{*}$ at the point of cessation of beam motion or when it is fully severed from the support. The fact that $\bar{\Pi}_{S}^{s}$ or $\beta$ increases monotonically with $I^{*}$ suggests that the damage criterion (Eq. 9) introduced by Shen and Jones [19] is applicable to distinguish between a mode II and III damage for a three-dimensional structural member. It is worth emphasizing that the critical $\beta$ value $\left(\beta_{c}=0.32\right)$ is obtained by calibration to the experimental data in [11] as mentioned in Section 2.2. With increasing $I^{*}$, deformation by shear and bending plays increasing important roles at the support, whilst the influence of membrane deformation on plastic work absorbed at the support reduces. Nonetheless, the majority of the plastic work dissipated at the support is by membrane deformation, apart for when $I^{*}>0.84$ in mode III where $\bar{\Pi}_{S}^{m}$ is smaller than either $\bar{\Pi}_{S}^{b}$ or $\bar{\Pi}_{S}^{s}$. In general, mode III deformation is governed by the combined effects of bending, membrane and transverse shear.

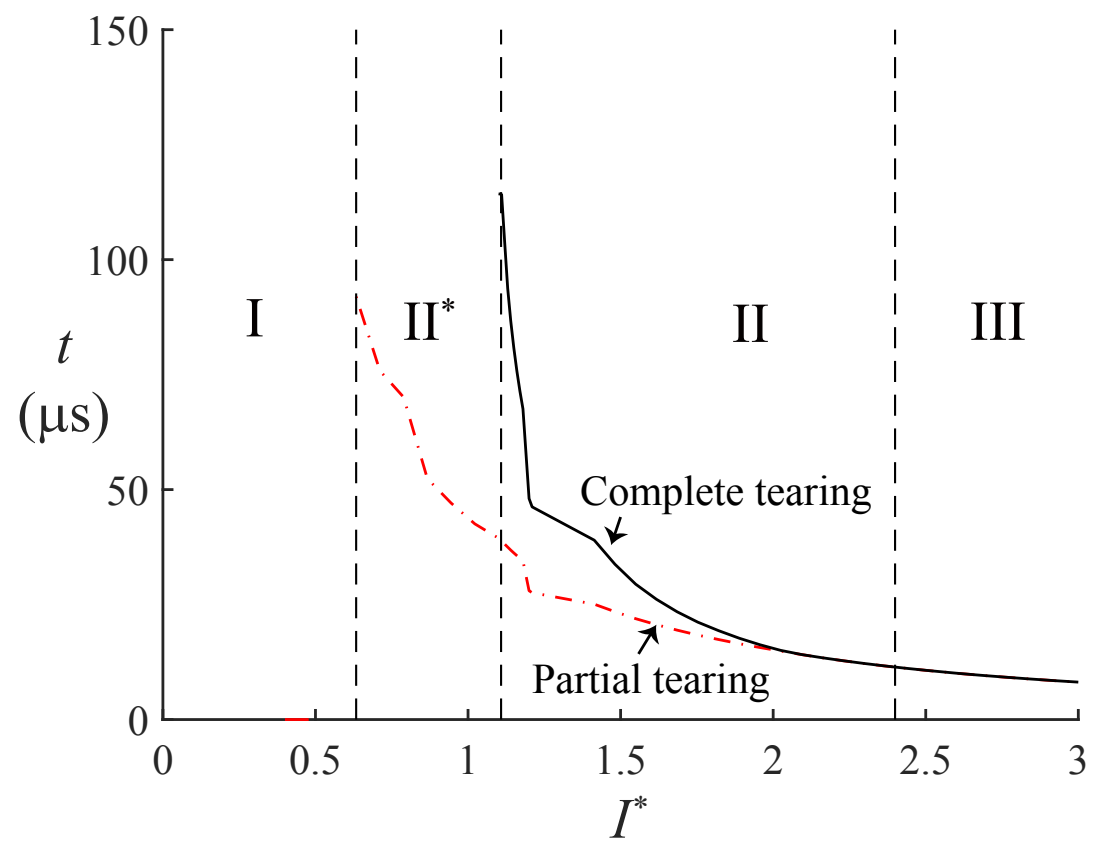

Figure 9: Time taken for partial and complete tearing against non-dimensional impulse $I^{*}$.

Figure 9 shows the time taken $t$ versus $I^{*}$ for partial tearing and complete detachment to occur. Notice that there is a monotonic decrease in $t$ for both curves with increasing $I^{*}$. The difference in time between the two curves after the transition from mode II* $\rightarrow$ II corresponds to the passage of time needed for tearing to progress from mid-point (points A in Fig. 2) at the support to the vicinity of boundary corner (points B in Fig. 2). This time difference is greatest at the transition between mode $\mathrm{II}^{*} \rightarrow \mathrm{II}$, after which it decreases monotonically with $I^{*}$. It is interesting to note that somewhere in mode II $\left(I^{*}>2.0\right)$ where the impulsive load becomes sufficiently intense, the two curves overlap, indicating instantaneous tearing along support. This agrees with the numerical prediction by Rudrapatna et al. [15].

To gain some insight into the differences in the damage of the plate for each mode of defor- 


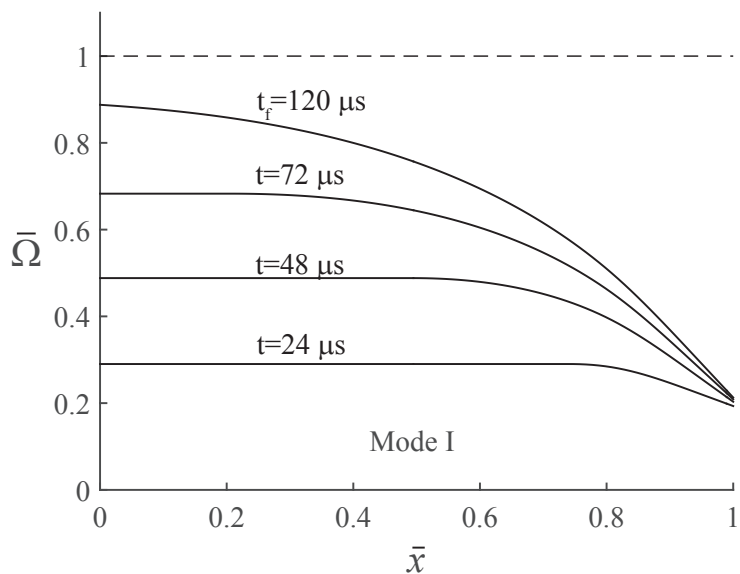

(a) $I^{*}=0.58$

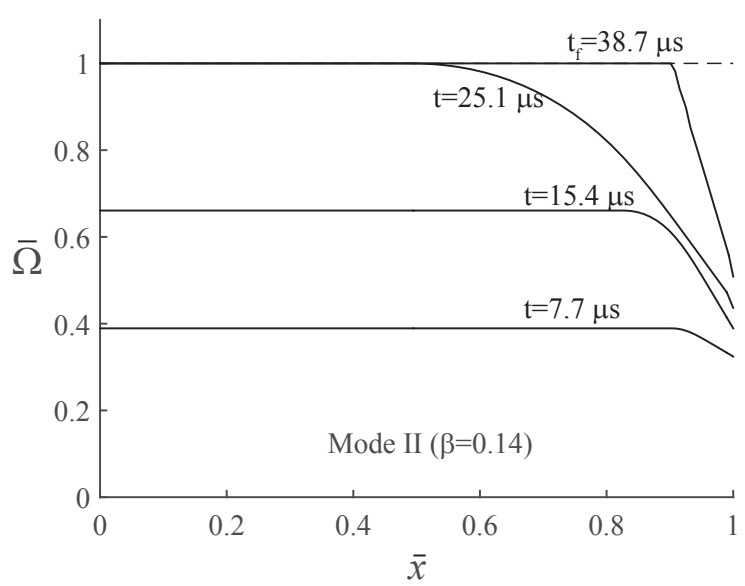

(c) $I^{*}=1.41$

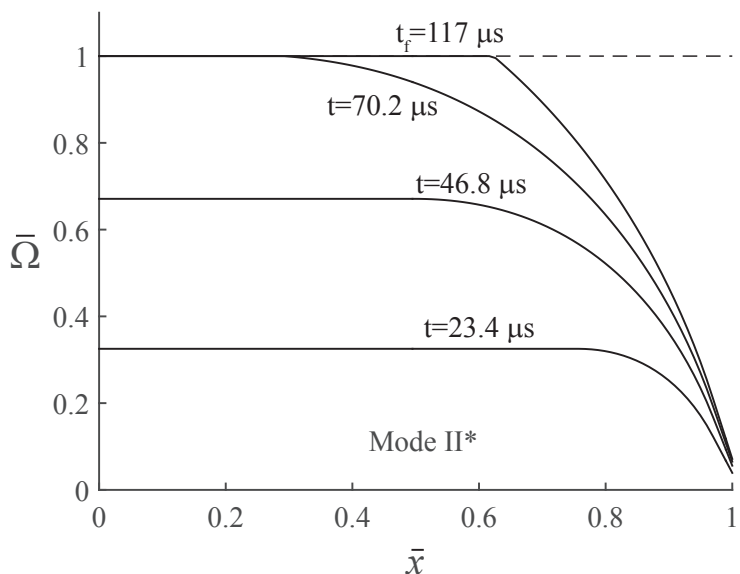

(b) $I^{*}=0.79$

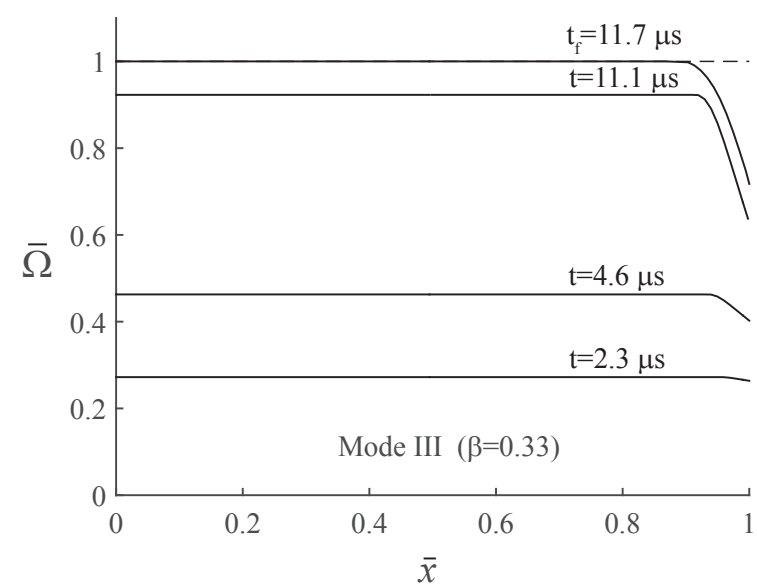

(d) $I^{*}=2.42$

Figure 10: Temporal variation of damage variable $\bar{\Omega}(x)$ along one side of the square plate at time $t_{f}$ : (a) mode I; (2) mode II*; (3) mode II; and (4) mode III.

mation, temporal variations of the non-dimensionalised density of plastic work dissipation are plotted in Figs. 10a-10d for modes I, II*, II and III respectively. It is evident in Fig. 10a that $\bar{\Omega}$ is greatest at the mid-point of the support and drops monotonically with $\bar{x}$ in mode I. Figures $10 \mathrm{~b}$ and $10 \mathrm{c}$ show that in modes II* and II tearing will progress from the central potion of the plate towards its corner where $\bar{\Omega}$ is below unity. Figure 10d shows an example of instantaneous tearing (i.e. damage criterion is met at both point $A$ and $B$ simultaneously) when complete detachment occurs when the impulsive load is greater than a critical value. 


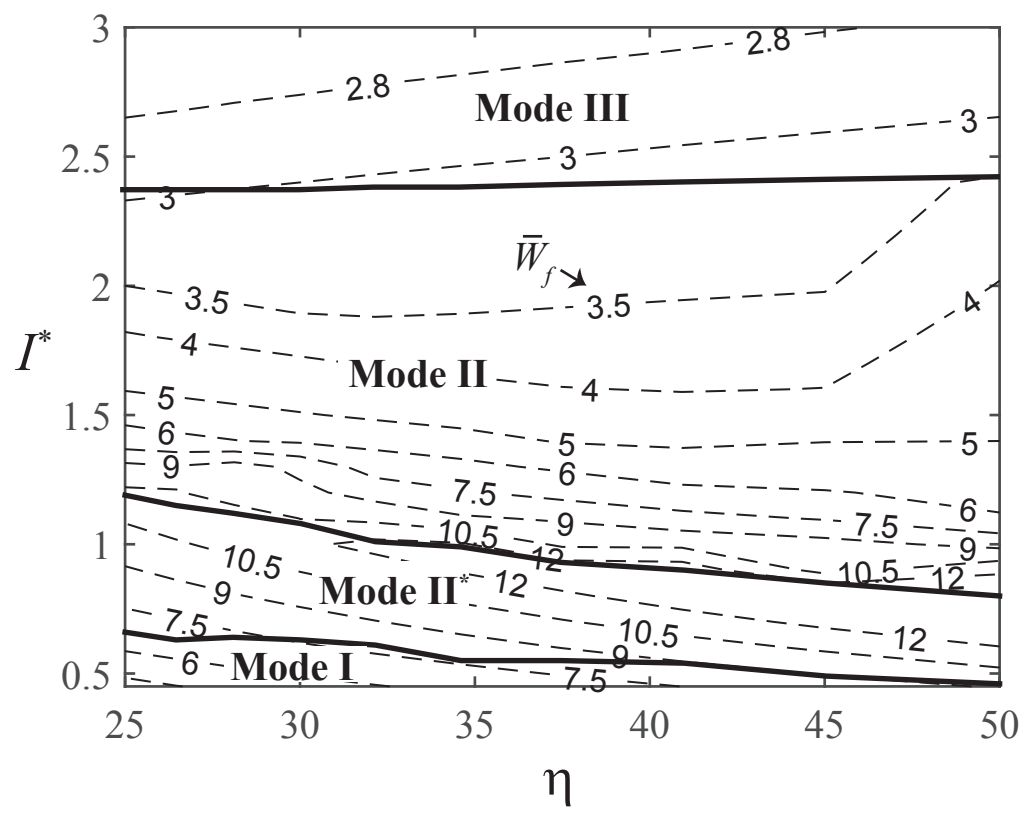

(a)

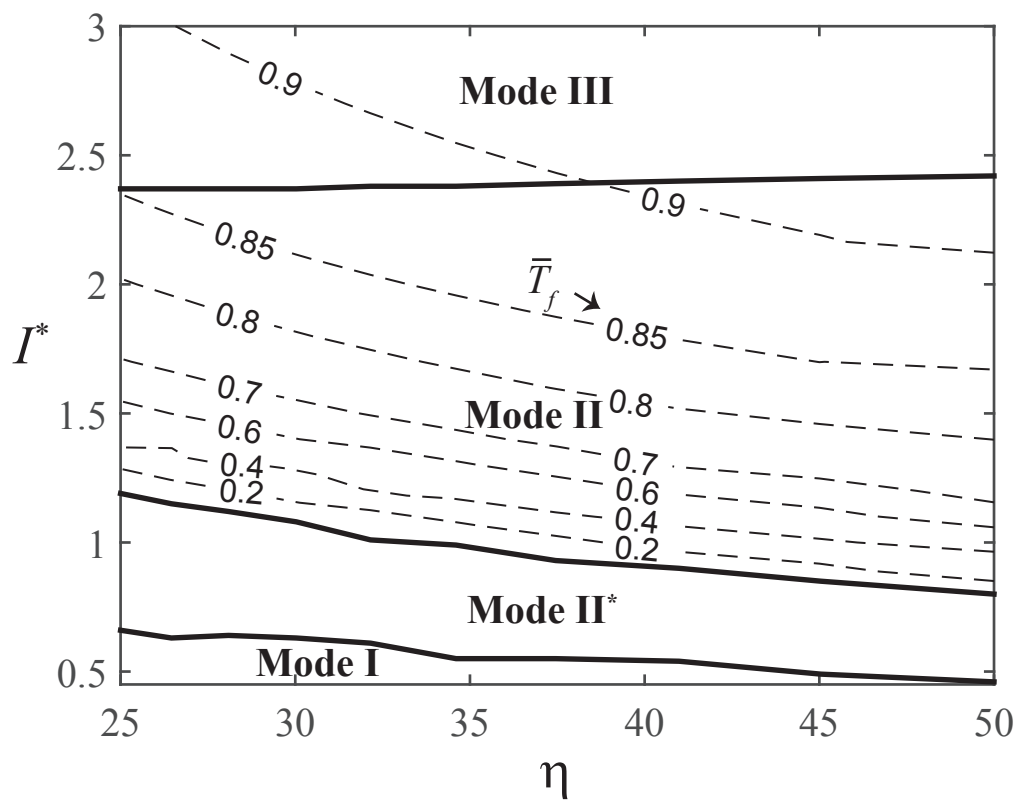

(b)

Figure 11: Design maps for square mild-steel plates (with a mass of $M_{P}=0.0992 \mathrm{~kg}$ ) where isolines correspond to a constant non-dimensional (a) transverse central deflection and (b) residual kinetic energy. 


\section{Blast resistant design}

The validated analytical model is employed here to construct design maps in $\eta-I^{*}$-space for practical ranges of $25 \leq \eta \leq 50$ and $0.45 \leq I^{*} \leq 3$. To ensure that the maps corresponds to plates of identical mass $M_{P}$, their half length to thickness ratio $(\eta)$ are varied according to $a=\left(M_{P} \eta / 4 \rho\right)^{1 / 3}$ and $h=\left(M_{P} \eta / 4 \rho\right)^{1 / 3} / \eta$. In this manner, the mass of the plate $\left(M_{P}=\right.$ $0.0992 \mathrm{~kg})$ remains a constant for different half length to thickness ratio $\eta$.

Figure 11a presents a map for square plates subjected to zero-period impulsive loading. It can be seen that the critical non-dimensional impulses at mode $\mathrm{I} \rightarrow \mathrm{II}^{*}$ and $\mathrm{II}^{*} \rightarrow \mathrm{II}$ transitions decreases monotonically with $\eta$. However, the critical $I^{*}$ needed to induce mode III deformation is insensitive to $\eta$. This agrees with the numerical results of [13] where it is suggested that the non-dimensional impulse at II $\rightarrow$ III transition does not vary with plate geometries and is essentially a material-related parameter. Isolines connecting constant values of transverse central plate deflection $\bar{W}_{f}$ within that particular mode of deformation are also inserted into the map. This gives a map which is useful to designers. Any pair of values of $\eta$ and $I^{*}$ now locates a point on the map. From the map, one can determine the deformation mode the plate deforms in and its mid-point deflection. Alternatively, it allows a designer to determine the critical non-dimensional impulsive $I^{*}$ delineating different modes of deformation and the corresponding central plate deflection, at a given half length to thickness ratio $\eta$. Another design map with isolines of non-dimensional residual kinetic energy for a fully-severed is plotted in Fig. 11b.

\section{Conclusions}

An analytical model is developed, and validated against experimental data and numerical predictions from existing literature, which is capable of predicting the deformation and damage associated with a fully clamped square mild-steel plate subjected to a wide range of impulsive loadings. Through the incorporation of an energy density damage criterion, the model is able to account for the combined effects of bending, membrane and transverse shear on ductile damage, and to capture partial and/or complete tearing at the support. Predictions by the analytical model is shown to be in good agreement with existing experimental results.

The analytical predictions offer the following insights: (1) Post-severance residual kinetic energy increases dramatically during mode II deformation and is considerable under a substantial zero-period impulse; (2) Membrane effects dominate the plate response in modes I, II* and II, whereas in mode III, bending, membrane and shear all play important roles; and (3) the time taken for both partial and complete tearing decreases monotonically with $I^{*}$, after a certain value of which they overlap, indicating instantaneous tearing along the entire support. 
It was shown that a thicker plate is able withstand higher impulses before the onset of partial and complete tearing, and the critical impulse needed to induce mode III deformation is insensitive to its length versus thickness ratio.

\section{Acknowledgment}

PJ Tan acknowledges the financial support by QinetiQ (Mr Robert Ball - Structures \& Survivability, Platform Design and Life Support IDT).

\section{References}

[1] Yuen, S. C. K., Nurick, G. N., Langdon, G. S., Iyer, Y., Deformation of thin plates subjected to impulsive load: Part iii: an update 25 years on, Int. J. Impact Eng. 107 (2017) 108-117.

[2] Aune, V., Fagerholt, E., Hauge, K., Langseth, M., Borvik, T., Experimental study on the response of thin aluminium and steel plates subjected to airblast loading, Int. J. Impact Eng. 90 (2016) 106 - 121.

[3] Aune, V., Valsamos, G., Casadei, F., Larcher, M., Langseth, M., Borvik, T., Numerical study on the structural response of blast-loaded thin aluminium and steel plates, Int. J. Impact Eng. 99 (2017) 131 -144 .

[4] Yuen, S. C. K., Butler, A., Bornstein, H., Cholet, A., The influence of orientation of blast loading on quadrangular plates, Thin-Walled Structures 131 (2018) 827-837.

[5] Stronge, W. J., Yu, T. X., Dynamic Models for Structural Plasticity, Springer, 1993.

[6] Yuan, Y., Tan, P. J., Shojaei, K. A., Wrobel, P., The influence of deformation limits on fluid-structure interactions in underwater blasts, Int. J. Impact Eng. 101 (2017) 9-23.

[7] Yuan, Y., Tan, P. J., Shojaei, K. A., Wrobel, P., On momentum transfer and external work done to clamped elasto-plastic beams in an air blast, Int. J. Mech. Sci. 146-147 (2018) 377-385.

[8] Menkes, S. B., Opat, H. J., Broken beams, Exp. Mech. 13 (11) (1973) 480-486.

[9] Teeling-Smith, R., Nurick, G., The deformation and tearing of thin circular plates subjected to impulsive loads, Int. J. Impact Eng. 11 (1) (1991) $77-91$.

[10] Olson, M., Nurick, G., Fagnan, J., Deformation and rupture of blast loaded square plates: predictions and experiments, Int. J. Impact Eng. 13 (2) (1993) 279 - 291.

[11] Nurick, G. N., Shave, G. C., The deformation and tearing of thin square plates subjected to impulsive loads an experimental study, Int. J. Impact Eng. 18 (1) (1996) 99 - 116.

[12] Nurick, G., Gelman, M., Marshall, N., Tearing of blast loaded plates with clamped boundary conditions, Int. J. Impact Eng. 18 (7) (1996) $803-827$.

[13] Yuan, Y., Tan, P. J., Deformation and failure of rectangular plates subjected to impulsive loadings, Int. J. Impact Eng. 59 (2013) 46 - 59.

[14] Gupta, N., Nagesh, Deformation and tearing of circular plates with varying support conditions under uniform impulsive loads, Int. J. Impact Eng. 34 (1) (2007) 42 - 59, international Conference on Impact Loading of Lightweight Structures.

[15] Rudrapatna, N., Vaziri, R., Olson, M., Deformation and failure of blast-loaded square plates, Int. J. Impact Eng. 22 (4) (1999) 449 - 467.

[16] Ramajeyathilagam, K., Vendhan, C., Deformation and rupture of thin rectangular plates subjected to underwater shock, Int. J. Impact Eng. 30 (6) (2004) 699 - 719.

[17] Jones, N., A theoretical study of the dynamic plastic behavior of beams and plates with finitedeflections, Int. J. Solids Struct. 7 (8) (1971) 1007 - 1029.

[18] Yu, T. X., Chen, F. L., The large deflection dynamic plastic response of rectangular plates, Int. J. Impact Eng. 12 (1992) 605-616. 
[19] Shen, W. Q., Jones, N., A failure criterion for beams under impulsive loading, Int. J. Impact Eng. 12 (1) (1992) $101-121$.

[20] Yuan, Y., Tan, P. J., Shojaei, K. A., Wrobel, P., Large deformation, damage evolution and failure of ductile structures to pulse-pressure loading, Int. J. Solids Struct. 96 (2016) 320 - 339.

[21] Shen, W. Q., Jones, N., Dynamic response and failure of fully clamped circular plates under impulsive loading, Int. J. Impact Eng. 13 (2) (1993) 259 - 278.

[22] Bodner, S. R., Symonds, P. S., Experiments on viscoplastic response of circular plates to impulsive loading, J. Mech. Phys. Solids 27 (1979) 91-113.

[23] Jones, N., Structural Impact, Cambridge: Cambridge University Press, 1989.

[24] Schiano-Moriello, D., Torii, R., Tan, P. J., On the post-blast residual length of a rectangular plate after severance, under preparation.

[25] Williams, J. H., Fundamentals of applied dynamic, New York: John Wiley \& Sons, 1996.

[26] Wen, H. M., Deformation and tearing of clamped work-hardening beams subjected to impulsive loading, Int. J. Impact Eng. 18 (4) (1996) 425 - 433.

[27] Alves, M., Jones, N., Impact failure of beams using damage mechanics: Part i: analytical model, Int. J. Impact Eng. 27 (8) (2002) $837-861$. 